\title{
Intelligent Wind-speed Monitoring System Based on the Zigbee Wireless Network
}

\author{
http://dx.doi.org/10.3991/ijoe.v11i1.4364 \\ Zhong liang $\mathrm{LU}^{1,2,3}$, Yu jiang $\mathrm{LI}^{1}$, Teng $\mathrm{FU}^{1^{*}}$ \\ ${ }^{1}$ Henan Polytechnic University, Jiaozuo, China; \\ ${ }^{2}$ Collaborative Innovation Center of Central Plains Economic Region for Coalbed/Shale Gas, Jiaozu, China; \\ ${ }^{3}$ The Collaborative Innovation Center of Coal Safety Production of Henan Province Jiaozuo, China
}

\begin{abstract}
In the use of traditional mechanical or electronic handheld anemometer, surveyors must measure wind speed in the prescribed measuring route, so it is easy to miss part of measurement or records, etc. The monitoring system uses CC2530 as the master controller, and calculates the value of wind speed by means of optical pulse emitted by an optical coupler. Different nodes are arranged on the wall of roadway, to set up a Zigbee network. When the surveyor's anemometer node transmit the measured value of wind speed to the fixed nodes of the roadway, the system will make use of the RSSI to calculate the coordinates of the surveyor, and data package be sent to the network coordinator and to the upper computer with the aid of optical fiber. The system can rapidly and accurately record the value of wind speed and the position of surveyors, to ensure the safety of surveyors.
\end{abstract}

Index Terms-Optical Pulse, Zigbee Network, Nodes, Receiving Signal Strength, Positioning

\section{INTRODUCTION}

In the measurement process of mine resistance parameters, the measured value of wind speed in the roadway is an important indicator. At present, most surveyors use mechanical anemometers, and the measured value of wind speed needs to calculate an average value before use, and a measuring error often occurs. KDF9002 [1] electronic anemometer developed by China Coal Technology Engineering Group Chongqing Research Institute uses 80C31 single-chip microcomputer as the master controller, and is externally connected to other hardware module. It can calculate the actual value to wind speed, but the chip's capability of data processing and reducing the power consumption of an anemometer still needs to further improve.

CC2530 is used the master controller of the wind-speed monitoring system, routing nodes are arranged on the wall of the roadway, to set up the Zigbee network [2-3]. If surveyors carry anemometer nodes, they will access to the network fast. The measured value of wind speed is transmitted to the network coordinator through wireless network, and to the upper computer with the aid of optical fiber, which is able to locate surveyors simultaneously. Measuring nodes use $3.7 \mathrm{~V}$ dry batteries for power supply, so its measurement accuracy, speed and reducing power consumption have been greatly enhanced, conductive to reduce wind-speed measuring error.

\section{The System StRUCTURE AND WORKING PRINCIPLE}

The system consists of anemometer node, network routing nodes, network coordinator, serial optical conversion module and upper computer. Anemometer node is a major part of the whole system, composed of impeller, optical coupler, signal processing circuits, CC2530 and LCD. The back of impeller blades is covered with white pigment, easy for reflection. Optical coupler installed $1 \mathrm{~cm}$ away from the rear of the impeller center is connected to other circuits. The system working process is shown in Figure. 1

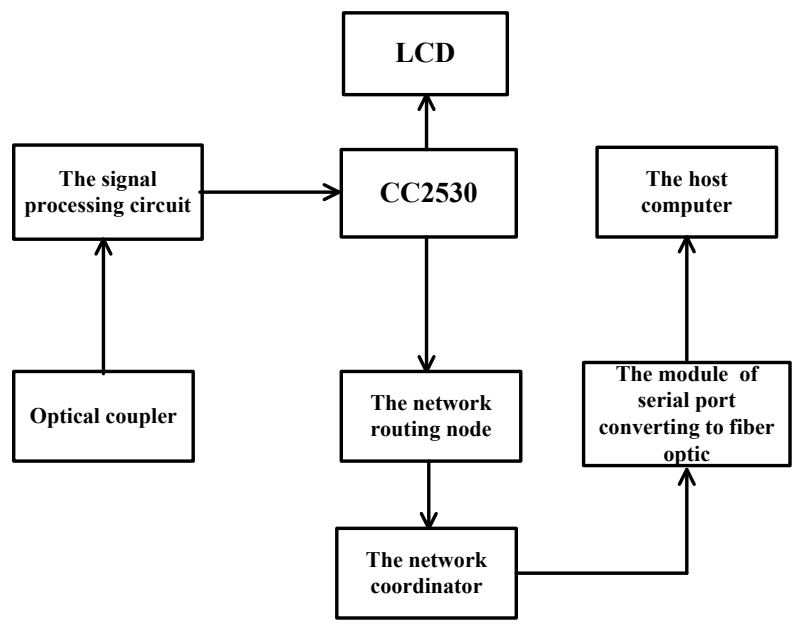

Figure 1. System working process

After arriving at the wind speed measuring point, surveyors first press the power switch, and then press the button of external interrupt. After a few seconds, if the LED indicator of anemometer node turns green, it indicates it accesses to the network successfully.CC2530 calculate the coordinates of surveyors by means of the RSSI, and then control relay module to switch on wind speed measuring circuit. At this moment, anemometer node starts to measure speed. When the impeller rotates with the action of horizontal wind, infrared light emitted from the optical coupler can be reflected discontinuously by white stripes on the blade, and the receiving terminal discontinuously receives infrared pulse, so that the circuit discontinuously outputs pulse [4-6]. Wind-speed momentum is converted into a series of electrical impulses by signal processing circuits, and CC2530 calculate the value of wind speed by measuring pulse frequency. Starting from external interruption, after the system timer counts down $1 \mathrm{~min}$, anemometer node automatically transmits the value of wind speed and the positioning information of surveyors to the network routing nodes, and then to the network coordinator. If the network coordinator is mounted with UART port, with the aid of serial optical conversion module, data will be transmitted to the upper computer eventually. 


\section{HARDWARE DESIGN}

The circuit diagram for anemometer node of the system is shown in Figure. 2, including $3.7 \mathrm{~V}$ dry batteries, 3.6V-to-5V circuit, HK4100F relay, wind-speed measuring circuit, CC2530, crystal oscillation circuit, radio frequency circuit, reset circuit, external interruption circuit, serial port-to- USB interface circuit, LCD display circuit, etc.

CC2530 [7-8] has 256KB flash memory and 20KB erasure cycles, 8KBRAM, programmable output power of up to $+4 \mathrm{dBm}$. In the power-down mode, when only the sleep timer runs, it has a current loss of less than $1 \mathrm{uA}$. In the external interruption mode, it has only current loss of $0.4 \mathrm{uA}$, It adopts dry batteries for power supply, to really ensure low power consumption and high efficiency running.

ITR20001voltage signal output by wind speed measuring circuit is converted into standard square wave signals through a voltage comparator composed of LM311, so that CC2530 can measure frequency conveniently.

Serial port-to-USB interface circuit consists of PL2303 chip and other peripheral circuit. With the help of the circuit, anemometer node can be directly linked with the computer for data transmission.

3.6V-to-5V circuit is comprised of MC34603 chip, and provides $5 \mathrm{~V}$ for wind speed measuring circuit. The circuit diagram is shown in the Figure 3.

\section{Wind SpeEd MEASUREMENT ANd Positioning PRINCIPLE}

\section{A. Principle of Wind Speed Measurement}

In the condition of low wind speed, the friction cannot be ignored, and the computational formula for start-up wind speed is shown in Formula (1). Therefore, at the low wind speed, rotational speed is not in a linear relationship with wind speed. In the condition of gale, wind speed is proportional to rotational speed:

$$
u_{\min }=\sqrt{B_{0} / 2 N}
$$

In the formula, Umin refers to start-up wind speed of the anemometer, $\mathrm{m} / \mathrm{min}$. B0 refers to static friction torque, N.m; N refers to blade logarithmic.

When friction torque is ignored, it can be obtained:

$$
n=k u / 2 \pi r
$$

In the formula, $\mathrm{n}$ refers to rotational speed of the impeller, $\mathrm{m} / \mathrm{min}$; $\mathrm{K}$ refers to fixed parameters of the impeller; $\mathrm{U}$ refers to the actual value of wind speed, $\mathrm{m} / \mathrm{min}$; r refers to radius of the impeller, $\mathrm{cm}$.

CC2530 can count the number of pulses within $1 \mathrm{~min}$, and the relationship between available frequency $f$ and rotating speed of the impeller $\mathrm{n}$ is as follows:

$$
n=f / 2 N
$$

Eventually, it is substituted into Formula (2):

$$
u=\pi r f / \mathrm{N} k
$$

\section{B. Positioning Principle}

Suppose the signal strength of emission node is known, based on the signal strength received by the receiving node, calculate the signal propagation loss, and then use the theory and empirical model to convert propagation losses into the distance value. Ultimately, calculate node

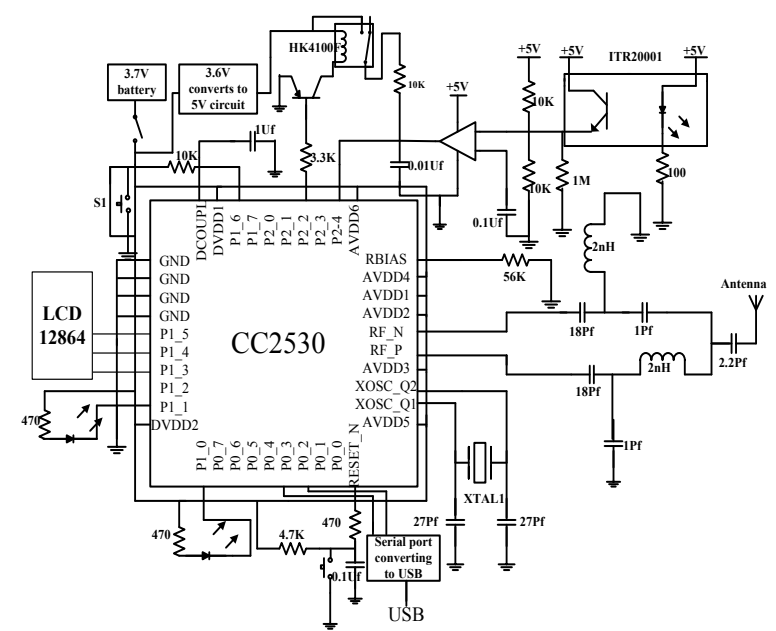

Figure 2. Anemometer node circuit

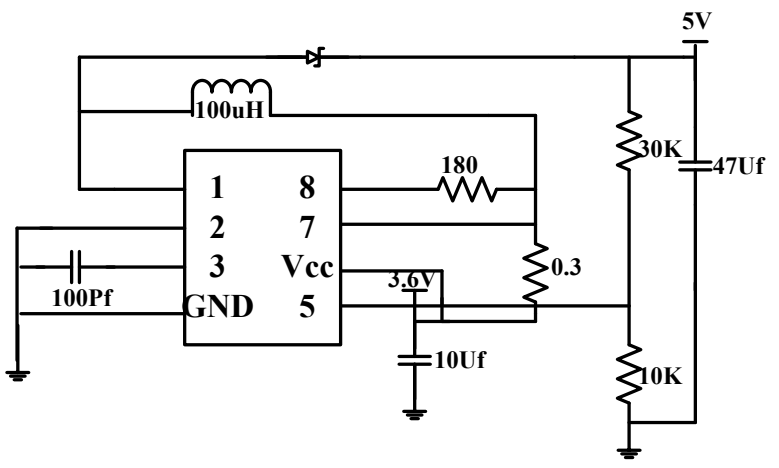

Figure 3. $33.6 \mathrm{~V}$-to-5V circuit

position according to the distance. The theoretical value of receiving signal strength can be shown as follows [9-11]:

$$
R S S I=-(10 n \cdot \lg (d)+A)
$$

In the formula: $\mathrm{n}$ refers transmission index; $\mathrm{d}$ refers to distance between routing node and wind speed node, m; A refers to receiving signal strength when the distance is $1 \mathrm{~m}$, dB.

At this time, the traditional least square method is used to modify the measured distance according to a particular circumstance. After the modified distance is obtained, the coordinates of anemometer node can be accurately estimated.

\section{SySTEM SOFTWARE DESIGN}

Tree network is used as the network, the program is initialized, and then protocol stack is initialized. Later, the program enters the application layer to handle the function $\operatorname{apsFSM}()$ and monitor Zigbee signal. When the routing node transmits a signal of joining the network, after the network coordinator makes a response, the routing node LED indicator turns green. Network addresses distributed by the routing node are $0 \times 0001,0 \times 0002,0 x 0003$, etc. successively. At this moment, press the button of anemometer node S1, if join the network successfully, the anemometer node LED indicator turns green, and the routing node continues to distribute long network addresses for the anemometer node. Meanwhile, the anemometer node sends RSSI sampling information. The anemometer node calls the function aplSendMSG() to transmit data to 
the network routing node after $1 \mathrm{~min}$, and to the upper computer with the help of Zigbee network. The anemometer node program flow chart is shown in the Figure 4.

Optical fiber is directly connected to the upper computer from the underground by means of optical serial conversion module. The upper computer can directly collect and monitor data via serial debugging assistant.

Port parameters are COM3, Baud rate of 115200, no check digit, 8 data bits and 1 stop bit.

\section{EXPERIMENTAL SIMULATION AND RESULT ANALYSIS}

In the experiment, the ground roadway simulation laboratory of Jiulishan Mine of Jiaozuo Coal Group is taken as the test site. The laboratory simulates the real environment of an underground roadway.

A section of $70 \mathrm{mX} 7.6 \mathrm{~m}$ roadway is selected. The electronic coordinatograph is used to initialize the settings of roadway position. Roadway length is set as $\mathrm{x}$ axis, and its width is set as y axis. 10 network modules are mounted on the roadway wall, and their ordinal numbers are 1 to 10 successively. No. 1-9 are set as routing nodes, No. 10 is set as the network coordinator. A serial optical conversion module is installed in the position $1 \mathrm{~m}$ away from the coordinator, the upper computer is placed outside the lab, and optical fiber is used to connect the middle. After the test starts, surveyors use the anemometer node to measure wind speed in nine measuring points as stipulated, and use the electronic coordinatograph to measure position coordinates at the same time. After completion of measurement, serial debugging assistant of the upper computer displays as shown in Figure. 5.

After comparing location information displayed on the upper computer with the measured coordinates, it shows that the system can accurately locate surveyors, and positioning accuracy is within $0.5 \mathrm{~m}$. After comparing the value of wind speed measured by the system with that measured by the traditional electronic anemometer, the precision has been improved greatly.

The system selects a section of straight roadway, so the attenuation degree of wireless signal is low, and data can be transmitted to the upper computer fast. In the real environment of down hole, wireless signal is affected by a complex environment, the phenomena of packet loss and long delay arise.

The system needs to improve in this aspect.

\section{CONCLUSIONS}

The system uses the Zigbee wireless network communication, and has the characteristics of low power consumption and fast speed. With the help of the upper computer, it can display the value of wind speed of anemometer node, the coordinates information of surveyors directly, accurately and rapidly. The system can quickly judge whether a part of measurement is missing, conductive to accurate measurement of mine resistance parameters.

\section{REFERENCES}

[1] Zhang Congli. "Development of KDF9002 mine anemometer". Coal Engineer,vol.10,no.1, pp. 1-3, 1994.

[2] Liu Zhigao, Li Chunwen, Geng Shaobo. "Blind zones personnel roadway network global positioning system". Journal of China Coal Society,vol.35(SO), pp. 236-242, 2010.

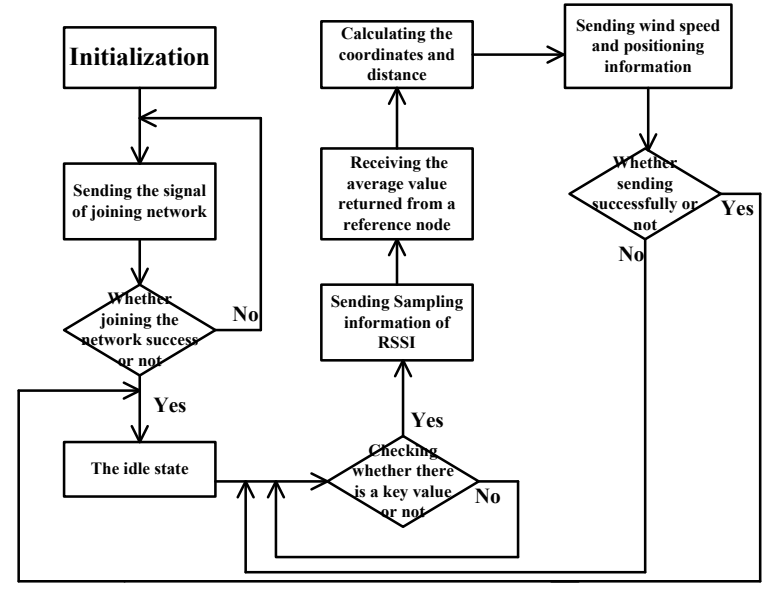

Figure 4. Anemometer node program flow

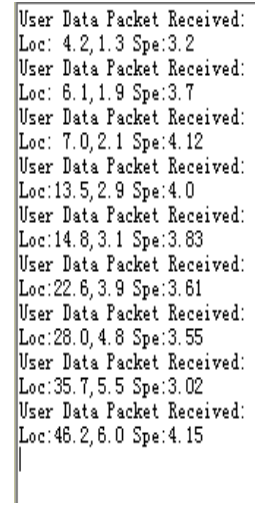

Figure 5. Upper computer monitoring software

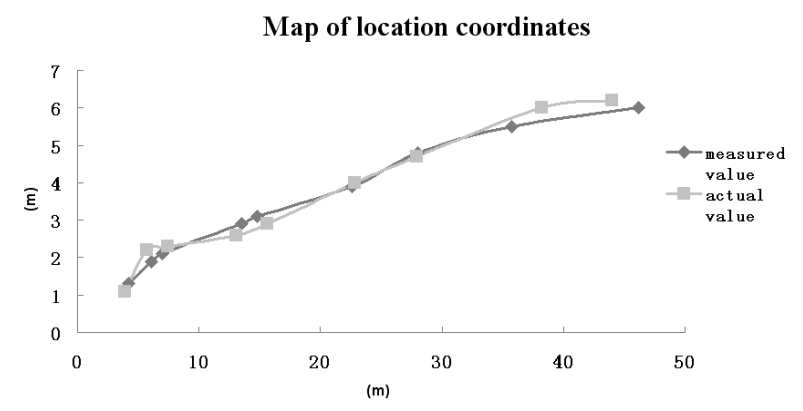

Figure 6. Positioning coordinate graph

[3] Qin Xianli,Fu Mingchao. "Mine intelligent portable anemometer based on Zigbee technology".Industrial Instrumentation And Automation Device, no.3, pp. 15-17, 2011.

[4] Niu Xiufan. "Portable wind measuring instrument". Changchun: Changchun Optical Precision Machinery And Physics Research Institute of China, pp. 26-30, 2011.

[5] Jin Jing, Tang Huiqiang. "Ultrasonic wind velocity measurement system based on ARM". Instrument Technique And Sensor,no 6, pp. 101-106, 2009.

[6] Han Yingtao,Ma Ruiqing,Zhou Qingmiao. "The equal precision frequency measuring method in speed rpm measurement display system application". Measurement And Control Technology,vol.17, no.1, pp. 53-55, 1998.

[7] CC2530 data sheet: system-on-chip for $2.4 \mathrm{GHZ}$ ZigBee/IEEE802.15.4[EB/OL].http://focus.ti.com/lit/ds/symlink/C C2530,July.11,2009.

[8] Zhai Lei,Liu Shengde,Hu Xianbin. "Zigbee Technology and Application". Beijing: Beihang University Press,2007. 
[9] Bai Xuhua, Zhang Ruifeng,Zhang Xiaomeng. "Design and implementation of indoor positioning system based on Zigbee network". Journal of Tianjin University of Technology,vol.4,no.2, pp. 12-14, 2012.

[10] Yang Wei,Zhou Siyong,Qiao Hua. "Coal mine safety monitoring wireless sensor network node positioning technology". Journal of China Coal Society,vol.32,no.6, pp. 652-656, 2007.

[11] Shen Zhiguang. "Underground personnel positioning system base station design and research based on Zigbee”. Jiaozuo:Henan Polytechnic University,pp. 45-52,2009.

\section{AUTHORS}

Zhongliang $\mathbf{L u}$ is a professor of School of Safety Science and Engineering, Henan Polytechnic University, Jiaozuo, 454003, China. He is also with the State Key Laboratory Cultivation Bas for Gas Geology and Gas Control at Henan Polytechnic University, as well as with the Collaborative Innovation Center of Central Plains Economic Region for Coalbed/Shale Gas, and the The Collaborative Innovation Center of coal safety production of Henan Province, Jiaozuo, China (email: zhonglianglu@126.com).

Yujiang Li, is a postgraduate of School of Safety Science and Engineering, Henan Polytechnic University, Jiaozuo, 454003, China (email: 313504782@qq.com).

Teng Fu, Corresponding author, is a postgraduate of School of Safety Science and Engineering, Henan Polytechnic University, Jiaozuo, 454003, China (email: futeng366@126.com).

Submitted 01 January 2015. Published as resubmitted by the authors 25 January 2015. 